\title{
Cardioprotection by PI3K-mediated signaling is required for anti-arrhythmia and myocardial repair in response to ischemic preconditioning in infarcted pig hearts
}

\author{
Feng Su ${ }^{1,2,4}$, Lan Zhao ${ }^{3,4}$, Shaoheng Zhang², Jiahong Wang², Nannan Chen², Qunlin Gong², Jinhui Tang², Hao Wang², \\ Jianhua Yao ${ }^{2}$, Qin Wang ${ }^{2}$, Ming Zhong ${ }^{1}$ and Jian Yan ${ }^{3}$
}

\begin{abstract}
Although the phosphatidyl-inositol-3-kinase (PI3K)/Akt pathway is essential for conferring cardioprotection in response to ischemic preconditioning (IP), the role of PI3K/Akt signaling in the infarcted heart for mediating the anti-arrhythmic effects in response to IP remains unclear. We explored the involvement of PI3K/Akt in the IP-like effect of connexin 43 and proangiogenic factors with particular regard to its role in protecting against ischemia-induced arrhythmia, heart failure, and myocardial remodeling. Groups of pigs were administered phosphate-buffered saline (PBS) or LY294002 solution.

Before induction of myocardial infarction (MI), pigs were grouped according to whether or not they underwent IP. Next, all animals underwent $\mathrm{MI}$ induction by ligation of the left anterior descending (LAD) coronary artery. Myocardial tissues from the pig hearts at 7 days after MI were used to assess myocardium myeloperoxidase and reaction oxygen species, infarct size, collagen content, blood vascular density, expression of Akt, connexin 43, and proangiogenic growth factors, using spectrophotometer, histology, immunohistochemistry, real-time RT-PCR, and western blot. At 7 days after MI, IP significantly reduced animal mortality and malignant ventricular arrhythmia, myocardial inflammation, infarct size, and collagen content, and improved cardiac function and remodeling; use of the PI3K inhibitor LY294002 diminished these effects. In parallel with a decline in Akt expression and phosphorylation by MI, LY294002 injection resulted in significant suppression of connexin 43 and proangiogenic factor expression, and a reduction of angiogenesis and collateral circulation. These findings demonstrate that the cardioprotective effects of IP on antiventricular arrhythmia and myocardial repair occur through upregulation of PI3K/Akt-mediated connexin 43 and growth factor signaling. Laboratory Investigation (2015) 95, 860-871; doi:10.1038/labinvest.2015.64; published online 1 June 2015
\end{abstract}

Sudden cardiac death comprises over $10 \%$ of all deaths from natural causes and constitutes a major health-related problem worldwide. In $\sim 80 \%$ of cases, sudden cardiac death is caused by sustained ventricular tachycardia (VT) and ventricular fibrillation (VF) secondary to acute myocardial infarction (AMI). ${ }^{1}$ Reducing malignant ventricular arrhythmia in infarcted hearts may be a key factor for improving prognosis of patients with MI. The strategy of myocardial repair using ischemic preconditioning (IP) provides an enticing option to reduce the severity of arrhythmias associated with AMI. Since its first description, myocardial IP has been found to enhance cardiac electrical stability in both animals and humans. However, the precise antiarrhythmic mechanisms underlying this adaptive process during ischemia are not well understood. It is generally thought that IP is able to limit the mass of myocardium at risk that ultimately becomes infarcted and results in reducing lethal arrhythmia. ${ }^{2,3}$ However, lack of IP enhanced inflammatory response, and subsequent left ventricle (LV) dysfunction, are related to VT/VF development after AMI. ${ }^{4}$ It has also been reported that cardioprotection with IP is related to the enhanced expression of angiogenic growth factors, such as vascular endothelial growth factor (VEGF) and basic fibroblast growth factors (bFGF) after MI. ${ }^{5,6}$ IP-induced protection also depends on functional connexin 43 (Cx43)-formed channels that play an essential role in IP of the heart, as Cx43 deficiency decreases myocardial

'Key Laboratory of Cardiovascular Remodeling and Function Research, Chinese Ministry of Education and Chinese Ministry of Public Health, Department of Cardiology, Qilu Hospital of Shandong University, Ji'nan, People's Republic of China; '2Department of Cardiology, Yangpu Hospital, Tongji University School of Medicine, Shanghai, People's Republic of China and ${ }^{3}$ Department of Cardiology, Dahua Hospital, Shanghai, People's Republic of China

Correspondence: Professor S Zhang, MD, PhD, Department of Cardiology, Yangpu Hospital, Tongji University School Of Medicine, 450 Tengyue Road, Shanghai 200090, People's Republic of China or Professor M Zhong, MD, PhD, Key Laboratory of Cardiovascular Remodeling and Function Research, Chinese Ministry of Education and

Chinese Ministry of Public Health, Department of Cardiology, Qilu Hospital of Shandong University, Ji'nan 250012, People's Republic of China.

E-mail: zhangsh67@yahoo.com or zhongmingzm@gmail.com

${ }^{4}$ These authors contributed equally to this work.

Received 28 November 2014; revised 18 March 2015; accepted 19 March 2015 
tolerance to ischemia/reperfusion injury and abolishes infarct size reduction induced by IP after MI. ${ }^{7}$ However, the exact underlying intrinsic relationship between $\mathrm{Cx} 43$ mediated protection, anti-inflammation, and angiogenesis on antiarrhythmic effects induced by IP remains to be established.

Accumulative evidence points to a role for phosphoinositide 3-kinase (PI3K)/Akt signaling as a final common pathway in the cardioprotective effect of IP. It was shown that use of a PI3K inhibitor diminished the protective effect of IP on infarct size and inflammation caused by ischemia/reperfusion. ${ }^{8}$ Phosphorylation of Akt leads to increased Akt activity and direct phosphorylation of $\mathrm{Cx} 43$, resulting in increased junctional size. ${ }^{9}$ Our previous study showed that blocking PI3K with LY294002 after permanent ligation of the coronary artery resulted in significant suppression of vascular VEGF, angiopoietin-1 (Ang-1), and bFGF expression, and a reduction in the amount of angiogenesis following endothelial progenitor cell transplantation in pigs. ${ }^{10}$ However, it is not clear whether the reduction in arrhythmias is a result of PI3K/ Akt-mediated anti-inflammation and angiogenesis or is due to $\mathrm{Cx} 43$-mediated protection of IP.

To investigate these questions, we used a porcine model of IP following MI induction by permanent ligation of LAD. The PI3K inhibitor LY294002 or phosphate-buffered saline (PBS) was injected into the left anterior descending (LAD) supply area before IP. Next, the animals randomly received IP following permanent ligation of LAD or direct ligation of LAD. This study was designed to explore the relationship between ventricular arrhythmia, heart function, cardiac remodeling, infarct size, collagen content, the expression of angiogenic cytokines, and angiogenesis following LY294002 injection or PBS. We also compared the effects of variations in these factors on myocardial protection induced by IP and determined whether PI3K/Akt signals are involved in the underlying mechanisms.

\section{MATERIALS AND METHODS}

An expanded Materials and Methods section containing details regarding animal allocation, animal model, study design, blood high-sensitivity C-reactive protein measurement, myeloperoxidase detection, coronary angiography, echocardiography, histology, quantitative real-time reverse transcription-PCR (qRT-PCR), and statistics is available in the Supplementary Data.

Animal Model, Study Design, and Ischemic Precondition Before MI preparation, pigs were randomly selected to receive an intramuscular injection of $5 \mathrm{ml}$ of PBS or LY294002, and then half were randomly chosen to receive IP. MI of miniswine was induced by ligation of the left anterior descending coronary artery.

\section{ECG Monitoring, Arrhythmia Recording, Defibrillation Protocol, and Survival Records}

ECG signals were monitored and recorded within $2 \mathrm{~h}$ after MI. Thereafter, ECG was recorded 1, 2, 3, 4, 5, 6, and 7 days after LAD ligation. Once ventricular tachycardia, ventricular flutter, or ventricular fibrillation occurred after LAD ligation, a 150 - or 200-J biphasic shock was given either asynchronously or synchronously. The numbers of survival animals were recorded in each group every day from the open-chest operation up to day 7 after LAD ligation (the end point).

\section{Coronary Angiography and Echocardiography}

Coronary angiography and echocardiography were performed in sedated mini-swine before MI, immediately after MI, and 7 days after MI.

Blood High-Sensitivity C-Reactive Protein Measurement Measurements of high-sensitivity C-reactive protein were performed on the COBAS Integra (Roche Diagnostics) with the $\mathrm{C}$-reactive protein-latex assay.

\section{Myeloperoxidase Detection}

Myeloperoxidase was quantified spectrophotometrically according to the manufacturer's instructions.

\section{Histology and Immunohistochemistry}

The size of the infarct was obtained by calculating the percentage of the infarcted area against the whole left ventricular area with the use of a digital image program (Scion Image J). The infarct zones, border zones, and remote zones from the autopsy specimens were embedded in paraffin, and were then stained by hematoxylin and eosin, Masson's trichrome stain, or immunohistochemistry.

\section{qRT-PCR and Immunoblotting}

Peri-infarct myocardial tissues were harvested and pulverized to extract RNA or protein for qRT-PCR and immunoblotting.

\section{RESULTS}

\section{Animal Survival}

A flow chart of the study protocol is shown in Supplementary Figure S1 online. In total, 90 experimental pigs were used in this study, 10 of which were randomly chosen to perform sham ligation and served as the control group for the measurement of baseline levels of PI3K/Akt-mediated cytokines and growth factors in cardiac tissue; the other 80 pigs underwent MI surgery. After open-heart surgery, 40 pigs were randomly divided into groups that either received a local intramyocardial injection of PBS or LY294002. After $30 \mathrm{~min}$ later, 20 pigs randomly received ligation of the LAD or IP and ligation of the LAD. During MI induction, IP without LY294002 injection significantly reduced the animal mortality caused by LAD ligation compared with previous LY294002 injection without IP (95\% in IPLI versus $70 \%$ in LYLI within 1 day after MI, $P<0.05$; Figure 1a), and this effect was sustained during the follow-up after MI preparation (95\% in IPLI versus $60 \%$ in LYLI at 7 days after MI, $P<0.01$; Figure 1a). There was no difference in survival function between the LYIPLI, LYLI, and LI groups. 

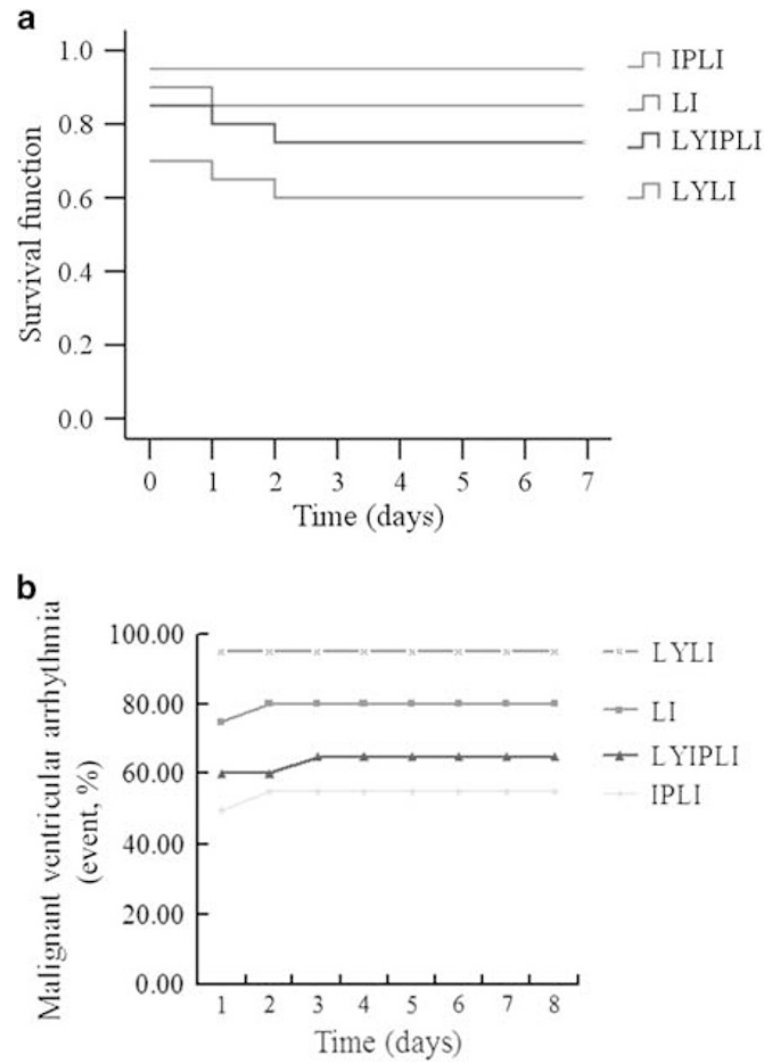

Figure 1 Animal survival rate (a) and malignant arrhythmia occurrence (b) ( $n=20$, each group). Groups are as follows: IP, ischemia preconditioning; LI, LAD ligation; LY, LY294002 injection.

\section{IP Reduced Malignant Ventricular Arrhythmia}

Malignant ventricular arrhythmia related to AMI occurred much more frequently in MI pigs that did not receive IP than in MI pigs that received IP, and was greatest in MI pigs receiving LY294002 injection without IP (95\% in the LYLI group versus $50 \%$ in the IPLI group within 1 day, $P<0.01 ; 95 \%$ in the LYLI group versus $55 \%$ in the IPLI group within 7 days, $P<0.01$ ), and greater in the LYLI group than in the LYIPLI group $(60 \%$ within 1 day, $P<0.01$; $65 \%$ within 7 days, $P<0.05$; Figure $1 \mathrm{~b}$ ). Detailed analysis showed that although LAD ligation led to VT or VF, IP significantly reduced VF time, lengthened the time to the first VF episode after permanent ligation, and decreased cumulative episode time. However, injection of LY294002 diminished these effects that were worst in the LILY group (Figures $2 a$ and $b$ ). Although there was no difference in the initial episode time between the LYLI group and the LYIPLI group, the arrhythmia episode times and cumulative episode time were greater in the LYLI group than those in the LYIPLI and the LI groups (Figure $2 \mathrm{~b}$ ). There was no animal death or malignant ventricular arrhythmia in the CON group (data not shown).

\section{IP Promoted Recovery of Infarcted Heart Function and Cardiac Geometry}

As shown in Figure 3, echocardiographic studies showed that before MI preparation, the baseline cardiac LVEF and LVFS function indexes in all five groups showed similar results. At 7 days after MI, the animals in the LYIPLI, IPLI, LYLI, and LI groups developed typical changes related to acute heart failure and LV structural remodeling compared with baseline levels and the control group. These changes included decreased LVEF (\%) and LVFS (\%), dilated LVEDV, LVESV, LVEDD, and LVESD, and thinned LVEDIVST and LVEDPWT. However, LVEF and LVFS in the animals that received IP-only treatment before MI (the IPLI group) were $6-12 \%$ and $10-$ $15 \%$ higher, respectively, than in the other groups $(P<0.01)$. In comparison, LVEF and LVFS in the animals not receiving IP treatment before MI (the LI group) were relatively low, and the LY294002 injection exacerbated the deterioration of these cardiac function indexes, leading to a significant or nearly significant decrease in LVEF and LVFS in the LYLI group. Consistent with the changes in LV cardiac function, LY294002 injection exacerbated cardiac structural remodeling processes in the LYLI group, including dilation of LVEDV, LVESV, LVEDD, and LVESD, and thinning of LVEDIVST and LVEDPWT. However, IP significantly ameliorated these indices, and the effect was found to be greatest in the IPLI group 7 days after MI. Interestingly, improvement in these indexes that resulted from IP was abrogated by injection of LY294002 in the LYIPLI group. There was no significant difference in LVEF, LVFS, LVEDIVST, and LVEDPWT between the LYIPLI group and the LI group, and between the LYIPLI group and the LYLI group, whereas LVEDV, LVESV, LVEDD, and LVESD were greater in the LYLI group than in the LYIPLI group and the LI group.

\section{IP Attenuated Myocardial Tissue Remodeling after MI}

In order to investigate the effects of IP on myocardial tissue remodeling after MI, myocardial inflammation, infarct size, and collagen content were observed by using $\mathrm{H} \& \mathrm{E}$ staining, triphenyl tetrazolium chloride staining (TTC staining), and Masson's trichrome stain. H\&E staining showed that compared with the control group, MI induced significant inflammation, including neutrophil infiltration, myocyte loss, and bleeding. This ischemic reaction was more serious in the LI pigs not receiving IP than in the IPLI pigs receiving IP alone, whereas adding injection of the PI3K inhibitor LY294002 exacerbated the reaction in the LYLI group and the LYIPLI group (Figure 4a). The quantitative indices for inflammation, hsCRP, MPO, and ROS were the highest in the LYLI group and were significantly decreased in the IPLI group (Figure $4 \mathrm{~b}$ ). In comparison, these inflammation indices were higher in the LYLI group than in the LYIPLI group and the LI group.

TTC staining showed that infarct size 7 days after MI was significantly smaller in the IPLI group than those in other groups (Figure 5a). By quantitative analysis, compared with ligation of LAD alone, IP resulted in a significant reduction in infarct size in the pigs 7 days after MI, and the effect was greatest in the IPLI group that received IP and not LY294002 injection. LY294002 diminished this IP effect, resulting in greater infarct size in the LYIPLI group and the LI group 
a

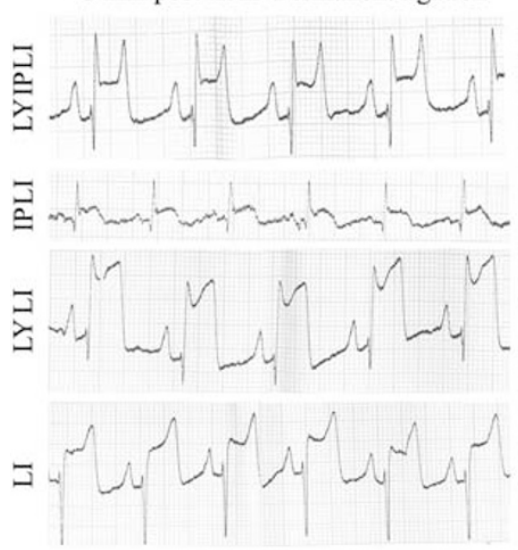

5-29 $\min$ LAD post-permanent ligation

30-45 min LAD post-permanent ligation

b
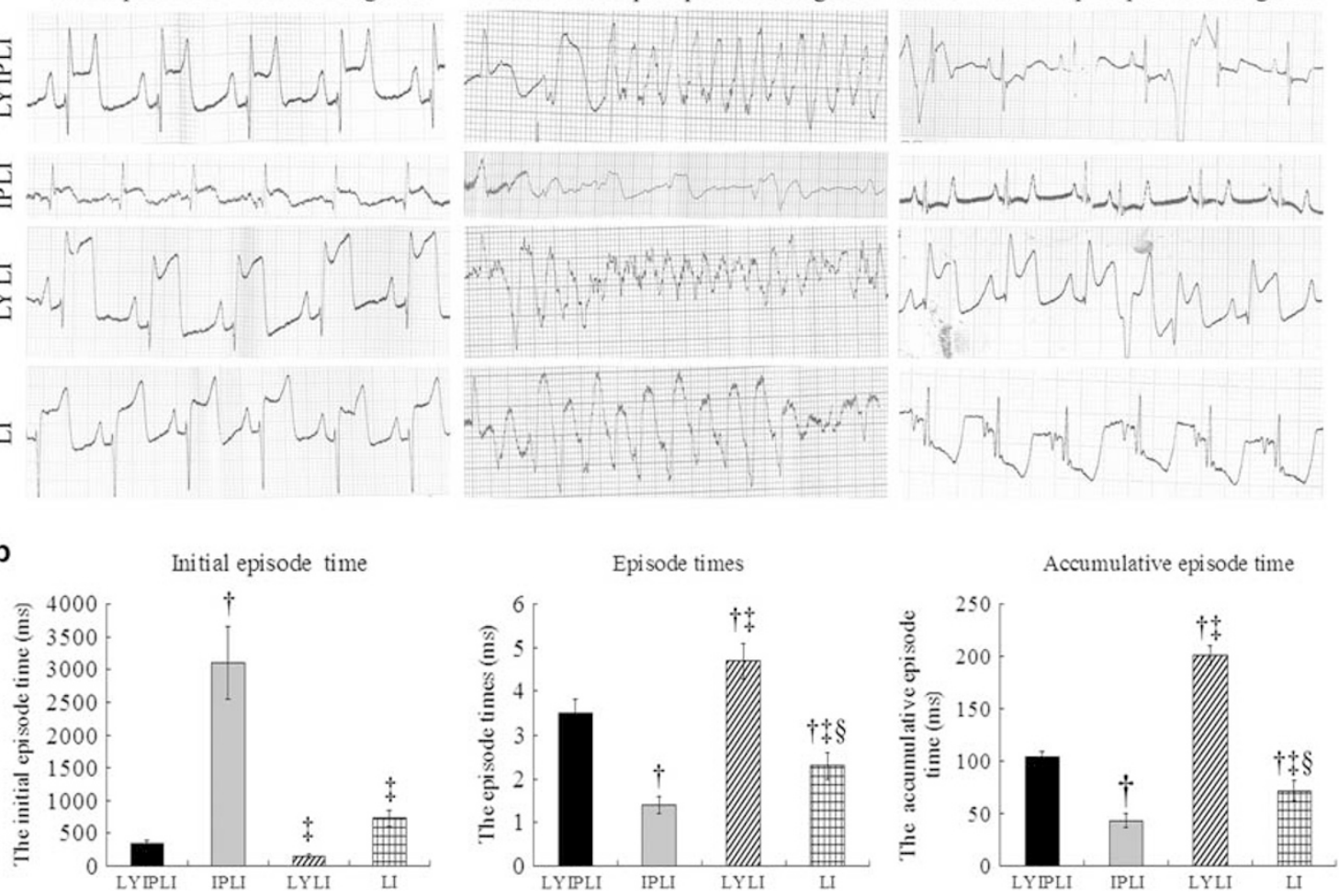

Figure 2 Occurrence of malignant ventricular arrhythmia in the various groups. (a) ECG before, during, and immediately after the first occurrence of ventricular fibrillation. (b) Statistical analysis of the interval period to the first episode of malignant ventricular arrhythmia, the episode times, and accumulative episode time within $12 \mathrm{~h}$ after permanent ligation. Data are expressed as mean \pm s.e.m. $P<0.05:{ }^{\dagger}$ versus $\mathrm{LYIPLI},{ }^{\ddagger}$ versus IPLI, ${ }^{\S}$ versus $\mathrm{LYLI}$ ( $n=10,15,19,12$, and 17 in the CON, LYIPLI, IPLI, LYLI, and LI groups, respectively). Groups are as follows: CON, control; IP, ischemia preconditioning; LI, LAD ligation; LY, LY294002 injection.

compared with the IPLI group. However, infarct size was greater in the LYLI group than in the LYIPLI group (Figure 5c).

As shown in Figure 5b, the collagen-rich myocardial scar in the infarcted wall stained blue, whereas viable myocardium stained red. Light microscopy revealed loss of cross-striations with appearance of focal inflammation and bleeding, and interstitial tissue edema in the LI group, which became more serious in LYLI pigs. In contrast, IP alleviated these pathological reactions. Moreover, greater salvaged myocardial tissue and smaller collagen were observed in the infarct zone of IPLI animals. Statistical analysis showed that there was significantly more viable myocardium in the IPLI group compared with the other three groups, with the lowest percentage in the LYLI group (Figure 5d). Collagen content was decreased within the MI regions in IPLI groups compared with the LI group; however, LY294002 injection increased collagen content, especially in the LYLI group, with a significant difference from the LI group (Figure 5e). Moreover, collagen content was greater in the LYLI group than in the LYIPLI group, and the viable myocardium in the infarct zone was smaller in the LYLI group than in the LYIPLI group. No differences were observed in border or remote zones among the four experimental groups.

\section{IP Enhanced Akt and Cx43 Expression in Ischemic Myocardium}

Real-time RT-PCR was performed to quantify the mRNA expression of Akt and $\mathrm{Cx} 43$ in peri-infarct border tissues. Compared with the control group, the highest expression level of Akt occurred in the IPLI group; this significantly declined in the LI group not receiving IP, and was lowest in the LYLI group (Figure 6a). Compared with baseline levels (control group), the lowest Cx43 mRNA levels occurred in MI pigs receiving LY294002 injection that did not receive IP, but significantly rose in MI pigs receiving IP but not LY294002 injections (Figure 6a). Immunoblots showed that protein levels of Akt and $\mathrm{Cx} 43$ were consistently higher in LI groups receiving IP compared with LI groups without IP, but injection of the PI3K inhibitor LY294002 significantly decreased these levels. Moreover, protein levels correlated well with mRNA levels (Figure 6b). Similar to Akt expression, both representative western blots with anti-Ser473 and antiThr408 antibodies and bar graphs of both Ser473 and Thr308 levels showed the greatest protein and phosphorylation levels in LI pigs receiving IP, but not LY294002 injection, and Akt pThr308 was significantly higher expressed in the LYIPLI group than in the LYLI group, and that the magnitude of 

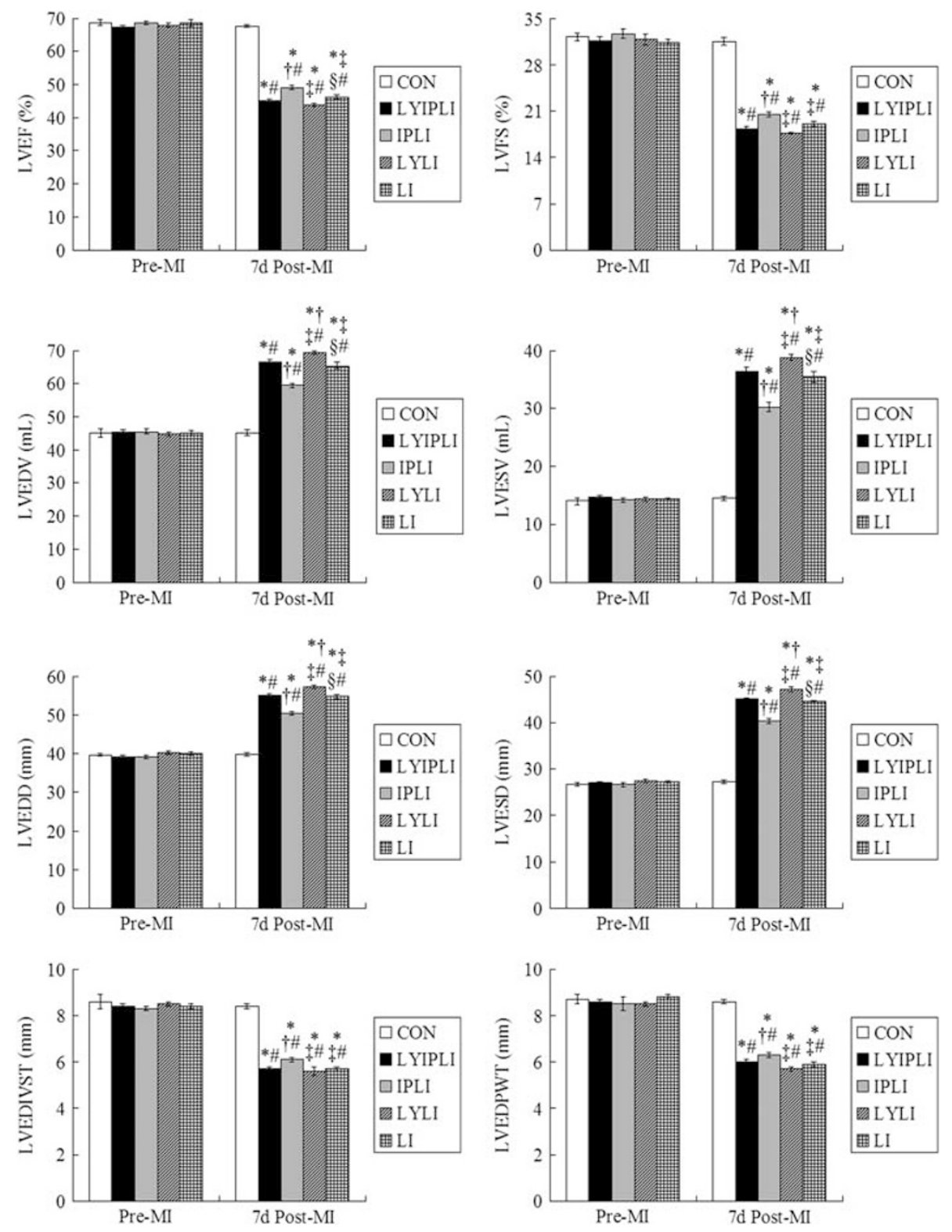

Figure 3 Changes in cardiac function and cardiac geometry induced by injection of LY294002, IP (ischemic preconditioning), and MI (myocardial infarction). Left ventricular ejection fraction (LVEF), LV fraction shortening (LVFS), LV end-diastolic volume (LVEDV), LV end-systolic volume (LVESV), LV end-diastolic interventricular septum thickness (LVEDIVST), and LV end-diastolic posterior wall thickness (LVEDPWT) were assessed by echocardiography before $\mathrm{MI}$ and 7 days after MI. $P<0.05:{ }^{*}$ versus CON, ${ }^{\dagger}$ versus LYIPLI, ${ }^{\ddagger}$ versus IPLI,,$^{\S}$ versus LYLI, " versus pre-MI. Data are expressed as mean \pm s.e.m. (pre$\mathrm{MI}, n=10$ in the control group, and $n=20$ each in the LYIPLI, IPLI, LYLI, and LI groups; 7 days after MI, $n=10,15,19,12$ and 17 in the CON, LYIPLI, IPLI, LYLI, and LI groups, respectively). See Figure 2 for abbreviation definitions.

pSer473 change was similar between the LYIPLI group and the LYLI group (Figure 6c). Similar Akt and Cx43 results were seen on immunohistochemical photomicrographs. Akt was mainly found in endothelial cells of capillary vessels and myocardiocytes, and $\mathrm{Cx} 43$ was mainly detected between myocardiocytes. Both Akt and Cx43 in both the infarct zone and the border zone were most highly expressed in the IPLI group, followed by the LI group, and significantly reduced in groups that had LY294002 injections (Figure 6d). The mRNA and protein expression of Akt and Cx43 were significantly lower in the LYLI group than in the LYIPLI group. No differences were observed in the remote zones among the four experimental groups (data not shown).

\section{IP Promoted Angiogenesis via Upregulation of Vascular Growth Factor}

Compared with the sham ligation groups (controls), permanent LAD ligation induced an overall increase in Ang1, bFGF, 
a

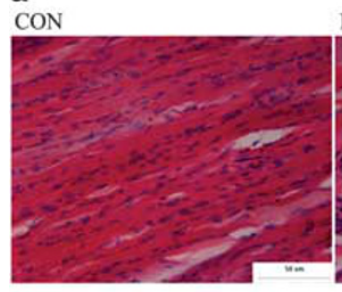

b

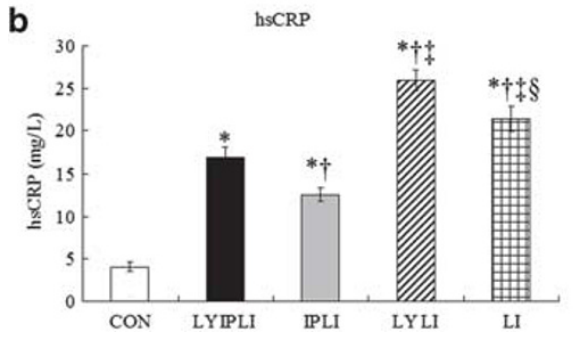

LY IPLI

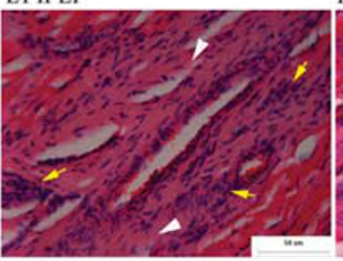

IPLI

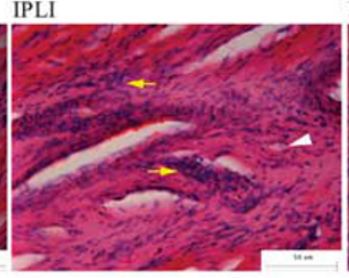

LYLI

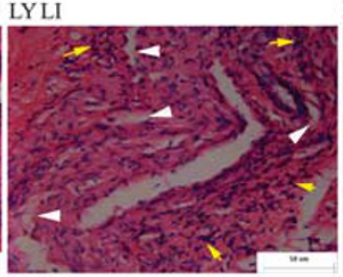

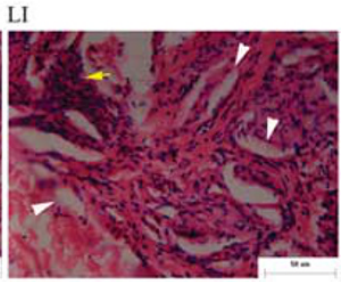

$\operatorname{ROS}$
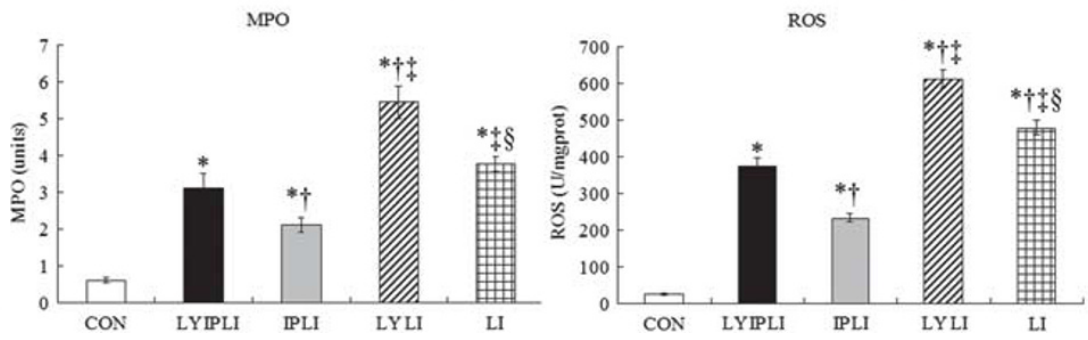

Figure 4 Analysis of inflammation in the infarcted myocardium of porcine at 7 days after infarct. (a) Representative H\&E staining under high magnification at 7 days after infarct. The yellow arrows and white arrowheads show inflammatory cell infiltration and myocyte necrosis, respectively. Scale bars $=50 \mu \mathrm{m}$. (b) Quantitative analysis of high-sensitivity C-reactive protein (hsCRP), myocardium myeloperoxidase (MPO), and reaction oxygen species (ROS) of individual groups 7 days after infarct. Data are expressed as mean \pm s.e.m. $(n=10,15,19,12$, and 17 in the CON, LYIPLI, IPLI, LYLI, and $\mathrm{LI}$ groups, respectively). $P<0.05:{ }^{*}$ versus CON, ${ }^{\dagger}$ versus LYIPLI, ${ }^{\ddagger}$ versus IPLI, ${ }^{\S}$ versus LYLI. See Figure 2 for abbreviation definitions.

and VEGF mRNA expression, and this augmentation was the greatest in IPLI pigs that received IP therapy before permanent ligation. The administration of LY294002 significantly reduced the increased levels of Ang-1, bFGF, and VEGF induced by ligation in both LYIPLI and LYLI pigs (Figures $7 \mathrm{a}-\mathrm{c}$ ). Western blot analysis showed the same change in protein expression in all groups (Figure 7d). Immunohistochemistry showed that Ang1, bFGF, and VEGF were detected in vascular endothelial cells as well as ischemic lesions, and the expression levels of these mediators were highest in IPLI pigs that had received IP therapy before LAD ligation, but expression levels of these mediators were significantly decreased in LYLI pigs that had received an LY294002 injection but not IP therapy before LAD ligation (Figure 7e). Tissue sections were stained for anti-factor VIII antibody (to detect endothelial cells), and the numbers of blood vessels were greater in the IP pigs that did not receive an LY294002 injection before LAD ligation compared with the LI groups receiving LY294002 injection and they were the highest in the IPLI pigs compared with the other groups (Figure $7 f$ and $g$ ). However, expression of Ang-1, bFGF, and VEGF and the number of blood vessels were greater in the LYIPLI group than in the LYLI group.

At 7 days after ligation, coronary angiography showed that there were some bridging collaterals in the distal occluded LAD from other blood vessels in IPLI groups (e.g., left circumflex artery, right coronary artery, or the diagonal branches of the proximal LAD), by which blood flow bypassed the occluded portion. Rentrop score was significantly higher in the IPLI group compared with the LI, LYIPLI, and IPLI groups, but LY294002 injection significantly reduced Rentrop scores in the LYIPLI and LYLI groups, and the
Rentrop scores were greater in the former than in the latter (Figure 7h).

Collectively, these findings suggest that the initial upregulation of PI3K/Akt-mediated proangiogenic cytokine expression is induced by myocardial ischemia, and IP therapy sustains this upregulation.

\section{DISCUSSION}

This study revealed several new and important findings. First, the infarcted porcine hearts with or without PI3K inhibitor LY294002, and with or without IP, exhibited a variety of ventricular arrhythmias and subsequent animal survival. At 7 days after MI, a decrease in mortality and infarct size predominated in porcine hearts receiving IP before MI, and use of the PI3K inhibitor LY294002 diminished this predominance. Second, individual differences in cardiac function and myocardial remodeling induced by permanent LAD ligation were caused by IP or LY294002 injection, demonstrating a link between malignant ventricular arrhythmia and changes in cardiac function and remodeling. Third, the PI3K inhibitor LY294002 resulted in a significant suppression of Cx43 expression and growth factors such as VEGF, Ang-1, and bFGF and a reduction in the amount of angiogenesis and collateral circulation. This illustrates the potential mechanisms underlying the observed cardiac protection of IP-induced reduction of malignant ventricular arrhythmia and cardiac repair.

\section{PI3K Inhibitor LY294002 Diminished IP-Induced Antiarrhythmias and Cardiac Repair}

In order to investigate the cardioprotection of IP, we started analysis with respect to three aspects: antiarrhythmia, heart function, and cardiac remodeling. Acute myocardial ischemia 
a

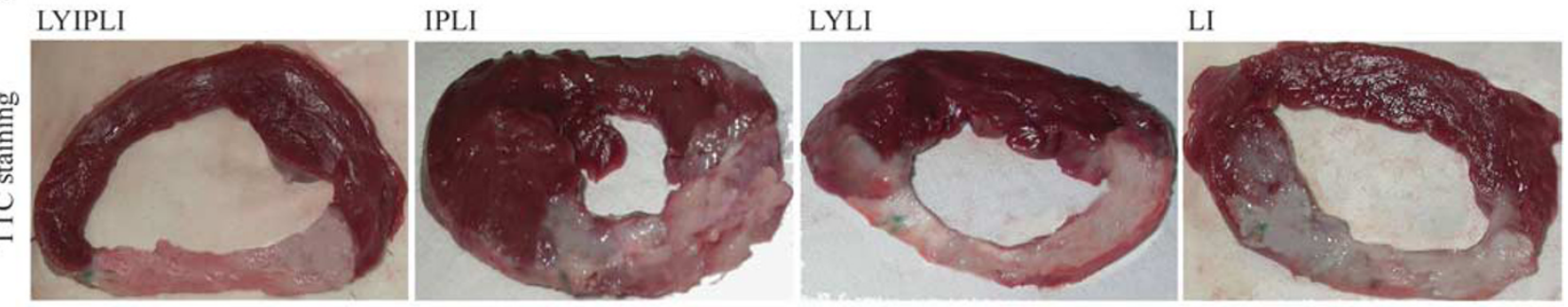

b

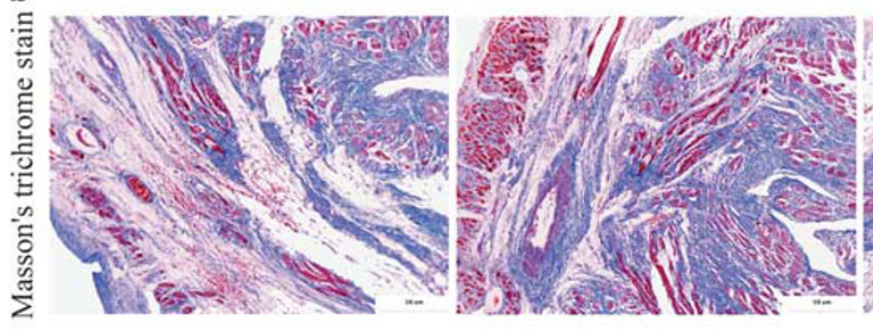

c

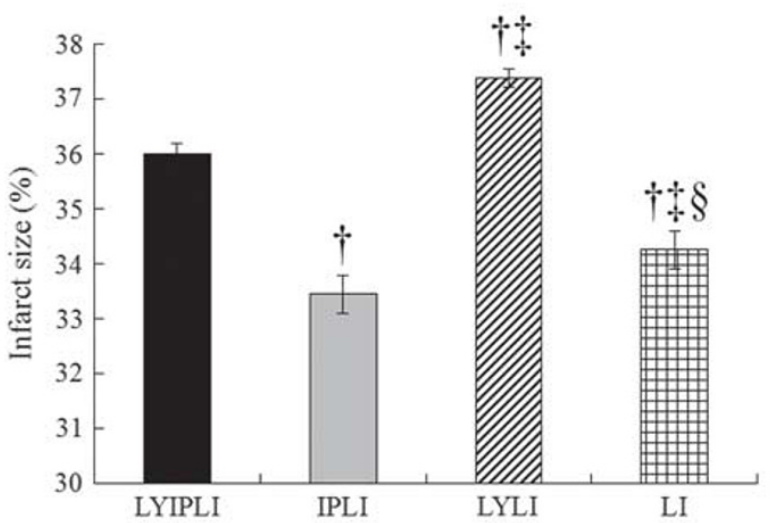

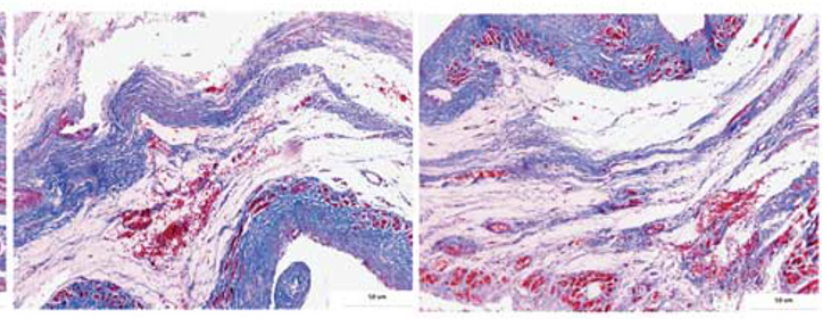

d

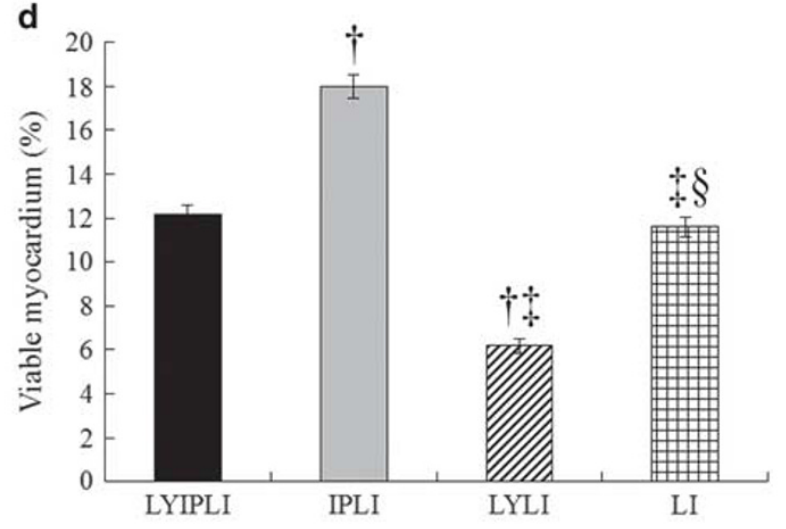

e

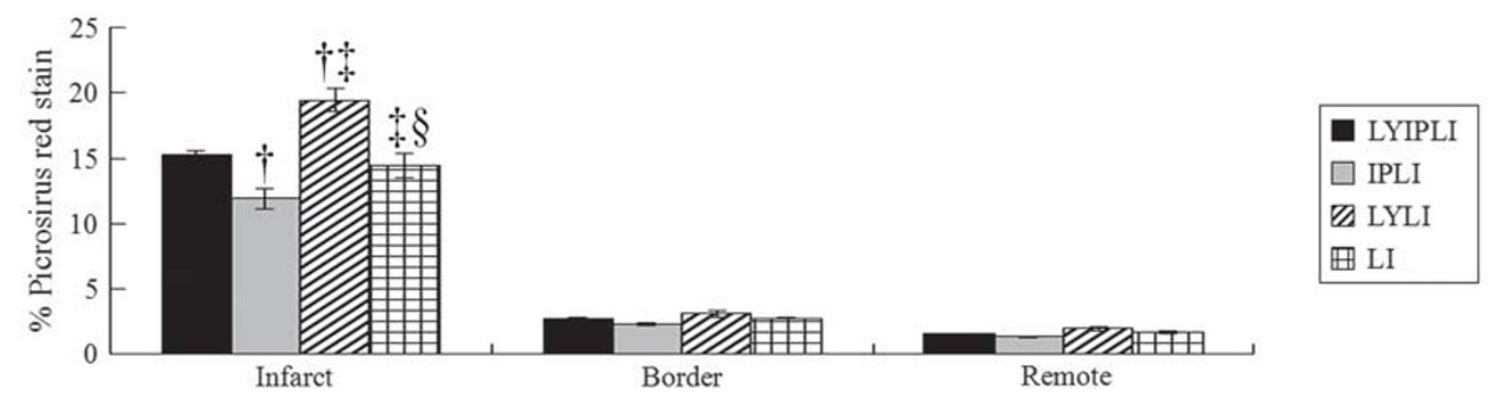

Figure 5 (a and b) Representative transverse myocardial sections stained by $\Pi \mathrm{CC}$ and Masson's trichrome stain of the LYIPLI, IPLI, LYLI, and LI groups 7 days after MI. Scale bars $=50 \mu \mathrm{m}$. None of the infarcted myocardium was stained red by TTC; the pale region is the infarcted myocardium. Viable myocardium is stained bright red. Fibrosis should be stained bright blue. (c-e) Quantitative analysis of infarct size, viable myocardium, and collagen content 7 days after Ml showed that significantly fewer fibrotic areas and infarct size and more surviving myocardium were seen in the IPLI group compared with the LI, LYLI, and LYIPLI groups, respectively. $P<0.05:{ }^{\dagger}$ versus LYIPLI, ${ }^{\ddagger}$ versus IPLI, ${ }^{\S}$ versus $\mathrm{LYLI}$. Data are expressed as mean \pm s.e.m. $(n=15,19,12$, and 17 in the LYIPLI, IPLI, LYLI, and LI groups, respectively). See Figure 1 for abbreviation definitions.

often results in malignant arrhythmia owing to both the direct effects of ischemia and the resultant hemodynamic disorder. ${ }^{11}$ In support of this idea, acute myocardial ischemia induced by only permanent ligation of LAD in this study in LI pigs brought about an increase in malignant ventricular arrhythmias; however, the hearts of IPLI pigs receiving IP before MI benefited from its antiarrhythmic effect: IP significantly reduced VF time, lengthened the time to the first VF episode after permanent ligation, and decreased the cumulative episode time (Figure 2). Injection of the PI3K inhibitor LY294002 
a

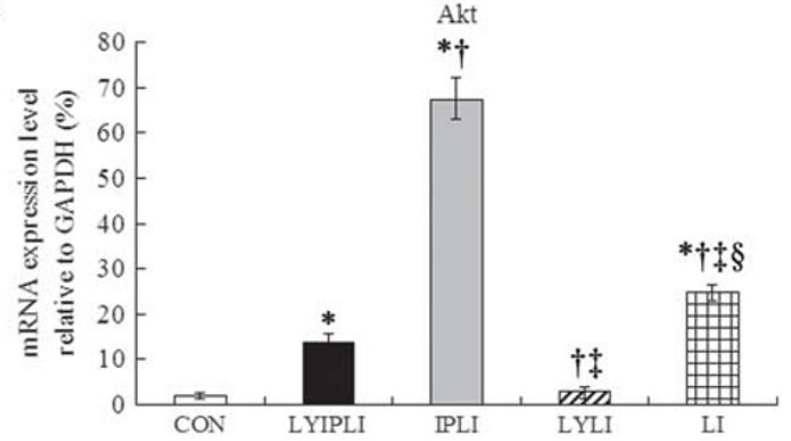

b

Akt

pSer473

pThr308

Connexin 43

GAPDH

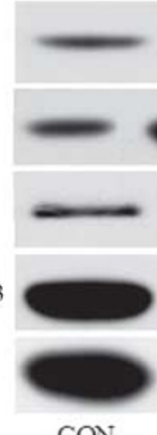

CON

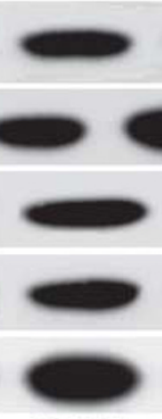

LYIPLI

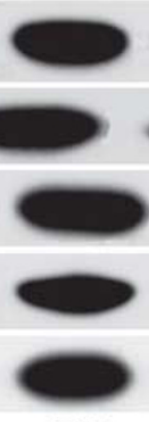

IPLI

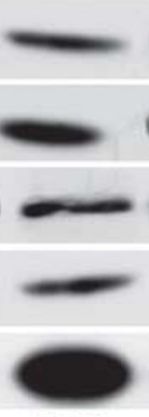

LYLI

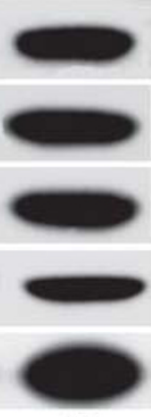

LI
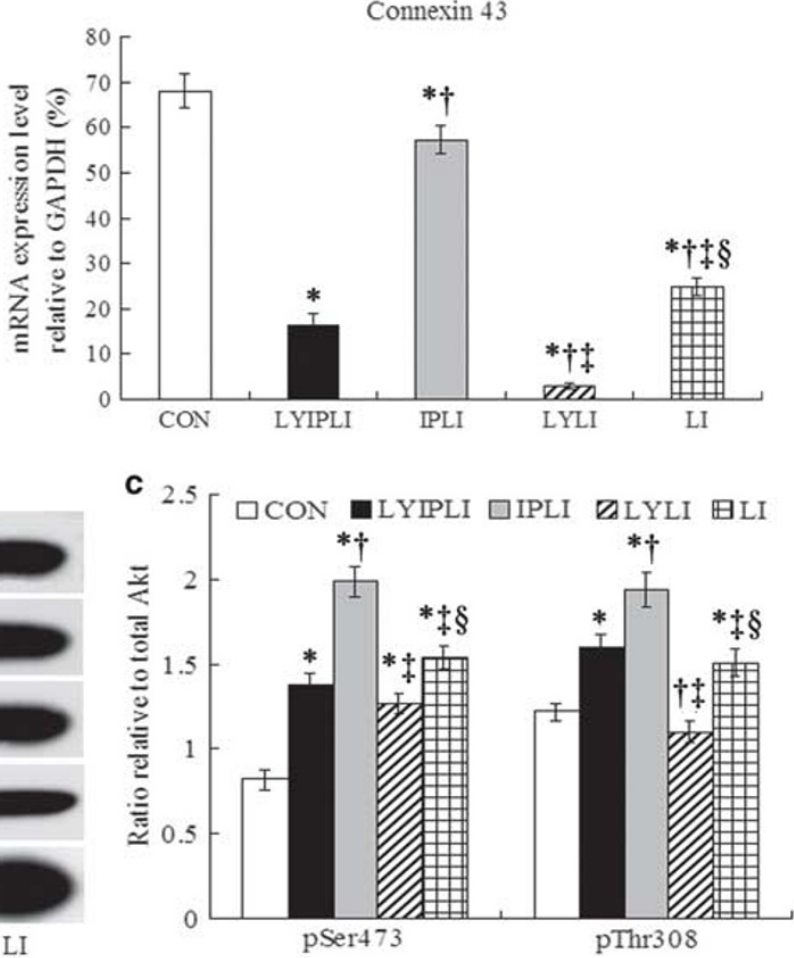

d $\mathrm{CON}$

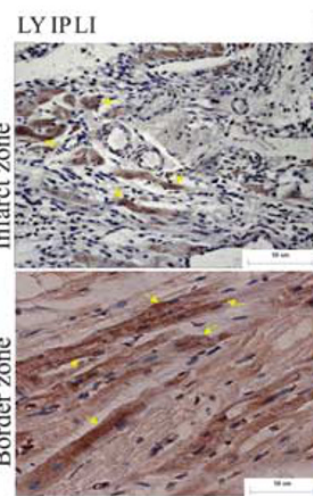

IPLI

LY LI

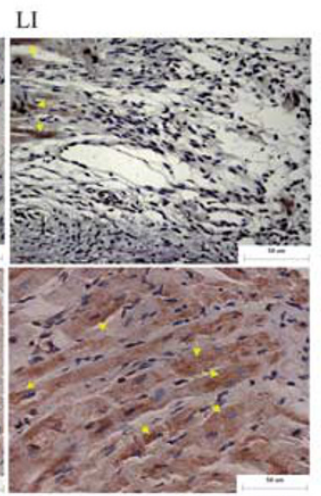

妾
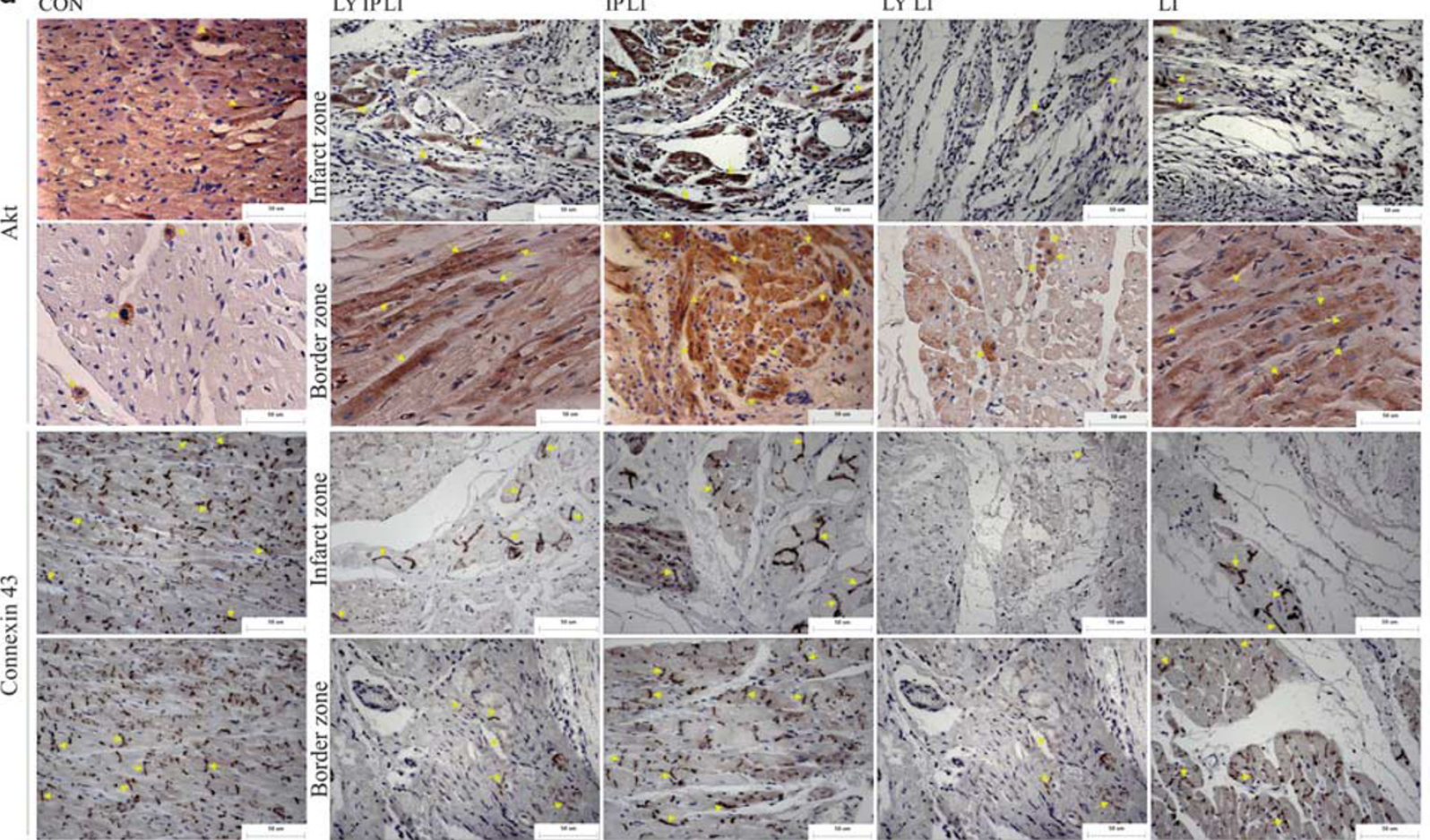

Figure 6 Akt and connexin 43 expression in the ischemic myocardium. (a) Quantitative analysis of Akt and connexin 43 at the mRNA level measured by reverse transcriptase-PCR (RT-PCR) in various groups. (b) Representative immunoblot electrophoresis showing the protein levels of Akt, phospho-Akt pAkt Ser473, pAkt Thr308, and connexin 43, respectively. (c) The ratio of phosphorylated Akt at Thr308 and Ser473/total Akt was calculated. (d) Local expression of Akt and connexin 43 at the infarct zone (the upper) and border zone (the lower) from the CON, LYIPLI, IPLI, LYLI, and LI groups, individually. Scale bars $=50 \mu \mathrm{m}$. Akt stained brown in the cytoplasm of myocardiocytes and blood vascular endothelium; connexin 43 also stained brown and was mainly expressed between myocardiocytes (yellow arrows). The nuclei of the cells peri-infarct were labeled with H\&E staining and appeared blue. Data are expressed as mean \pm s.e.m. ( $n=10,15,19,12$, and 17 in the CON, LYIPLI, IPLI, LYLI, and LI groups, respectively). $P<0.05:{ }^{*}$ versus control, ${ }^{\dagger}$ versus LYIPLI, ${ }^{\ddagger}$ versus IPLI, ${ }^{\S}$ versus LYLI. See Figure 2 for abbreviation definitions. 
significantly reduced the beneficial effects of IP in LYLI pig hearts and LYIPLI pig hearts. In accordance with these changes, infarct size was smaller and there was significantly more viable myocardium within the infarct zone in the IPLI group compared with the LI group, and injection of the PI3K inhibitor LY294002 significantly decreased residual viable myocardium, and enlarged infarct size in the LYIPLI and LYLI group (Figure 5). This result is corroborated by another study that examined Langendorff-perfused rat hearts, and showed that another PI3K inhibitor, wortmannin, blunted the antiarrhythmic and anti-infarct effects of IP. ${ }^{12}$ All these data indicate that the antiarrhythmogenesis induced by IP after MI is one of the main product of these beneficial changes that may be involved in the protection of residual myocardial viability and decrease in infarct size via activation of the PI3K/ Akt pathway. Even so, the findings in this study may not be enough to demonstrate that inhibition of PI3K directly inhibited the arrhythmias, and further works wait for performing analysis of arrhythmic risk and electrophysiological study that ensure us to obtain sufficient and appropriate direct evidences of IP-induced cardioprotection and the involvement of PI3K in antiarrhythmogenesis.

Another major finding of our study was that the IP-treated pigs exhibited significant cardioprotection from cardiac repair, including heart function recovery and improvement of LV remodeling. Heart function indices EF and FS were significantly higher in IPLI animals receiving IP-alone treatment before MI than in the LI groups not receiving IP, and the LY294002 injection decreased these cardiac function indices (Figure 3). Consistent with changes in LV cardiac function, LY294002 injection weakened the effects of IP on inhibiting LV structural remodeling in the LYLI group, as assessed by echocardiography, leading to dilation of LVEDV, LVESV, LVEDD, and LVESD, and thinning of LVEDIVST and LVEDPWT, compared with baseline levels and those of the control group at 7 days after MI (Figure 3). Xu et al ${ }^{13}$ made a similar discovery that intermittent hypobaric hypoxia preconditioning exerts a therapeutic effect on MI a

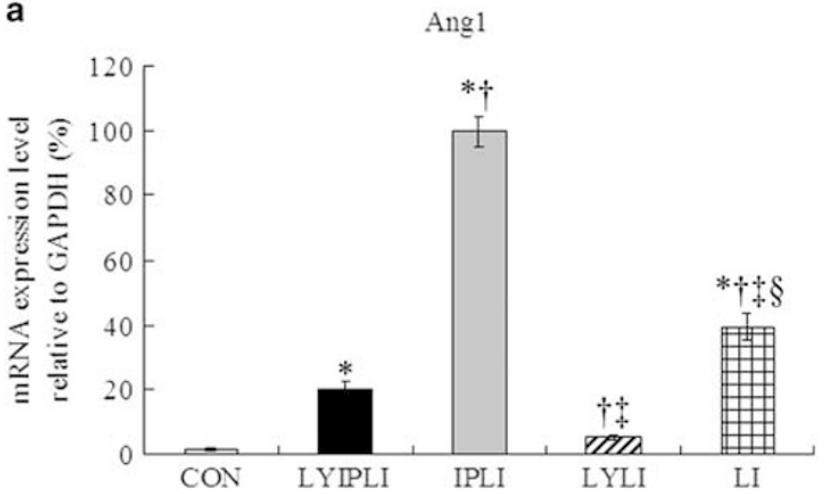

c

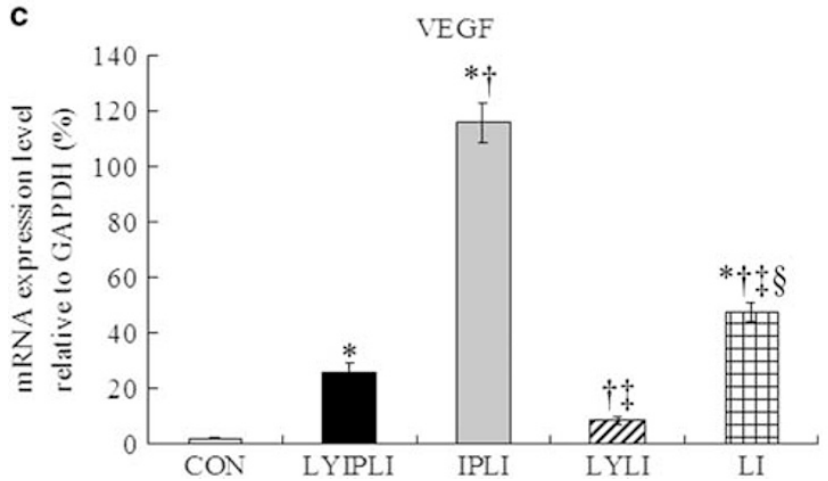

b

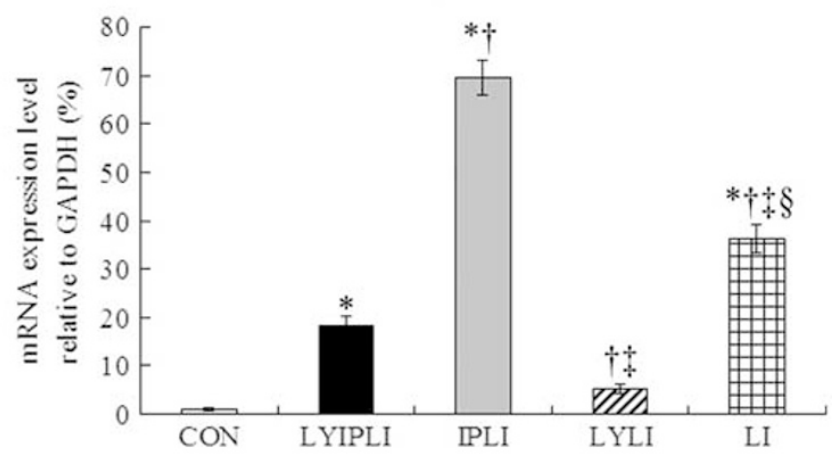

d

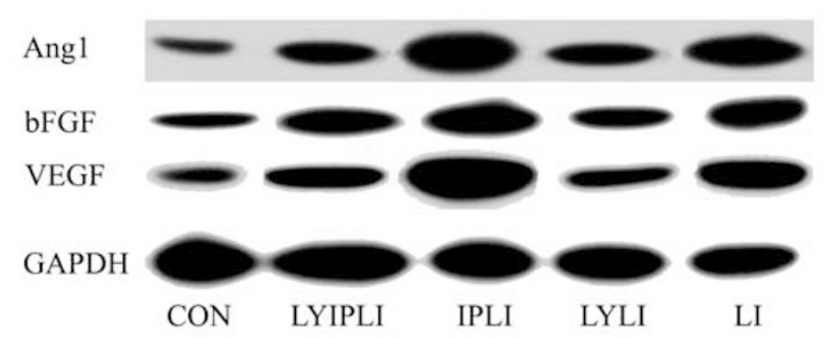

Figure 7 Angiopoietin-1 (Ang-1), basic fibroblast growth factor (bFGF), vascular endothelial growth factor (VEGF), and blood vascular density in the infarcted porcine hearts 7 days after MI. (a-c) Quantitative analysis of Ang-1, bFGF, and VEGF mRNA levels measured by quantitative real-time reverse transcription-PCR. (d) Representative western blots of Ang-1, bFGF, and VEGF protein expression within the left ventricular peri-infarct areas at 7 days after LAD ligation. Protein expression correlated well with mRNA expression. (e and f) Immunohistochemistry staining shows local expression of Ang-1, bFGF, VEGF, and factor VIII in the peri-infarct areas from the CON, LYIPLI, IPLI, LYLI, and LI groups 7 days after MI in the porcine hearts, respectively. Scale bars $=50 \mu \mathrm{m}$. Ang-1, VEGF, bFGF, and factor VIII stained brown (cytoplasm-stained brown). The number of blood vascular density images assessed by immunohistochemistry were greatest in the peri-infarct areas of pigs receiving IP but not LY294002 injection before LAD ligation. Scale bars $=50 \mu \mathrm{m}$. ( $\mathbf{g}$ and $\mathbf{h}$ ) Quantitative data of the number of blood vessels and Rentrop scores in various groups 7 days after Ml. All data are expressed as mean \pm s.e. m. $\left(n=10,15,19,12\right.$, and 17 in the CON, LYIPLI, IPLI, LYLI, and LI groups, respectively). $P<0.05:{ }^{*}$ versus CON, ${ }^{\dagger}$ versus $\mathrm{LYIPLI},{ }^{\ddagger}$ versus IPLI, ${ }^{\S}$ versus $\mathrm{LYLI}$, respectively. See Figure 2 for abbreviation definitions. 
e
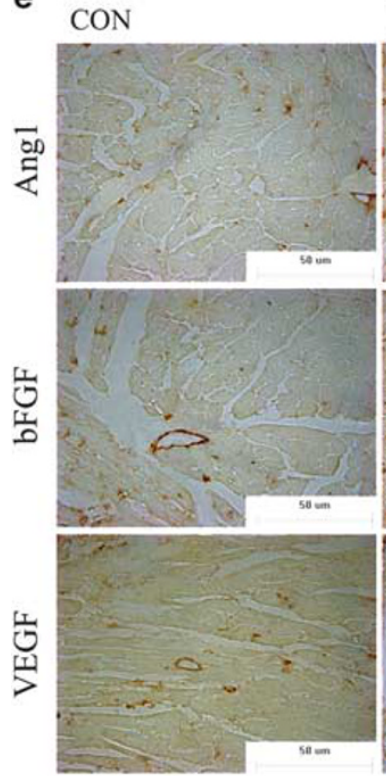

f

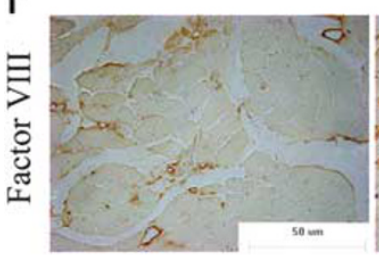

g

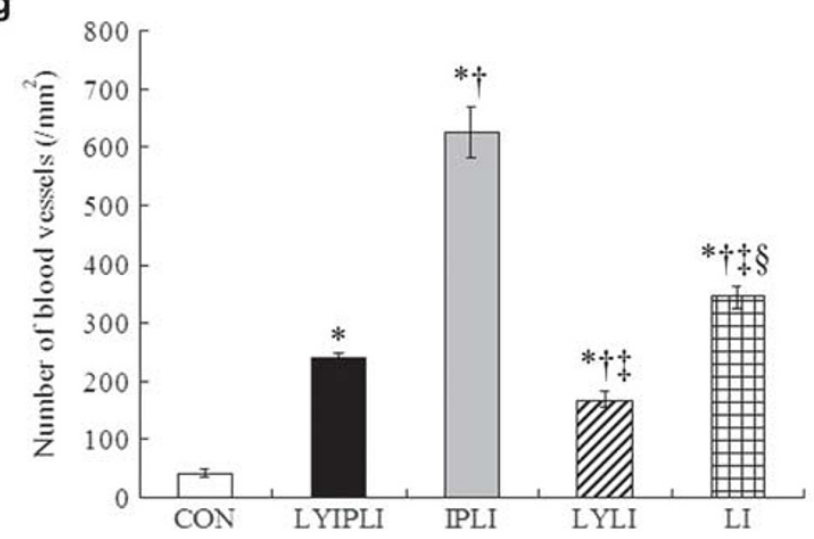

IPLI
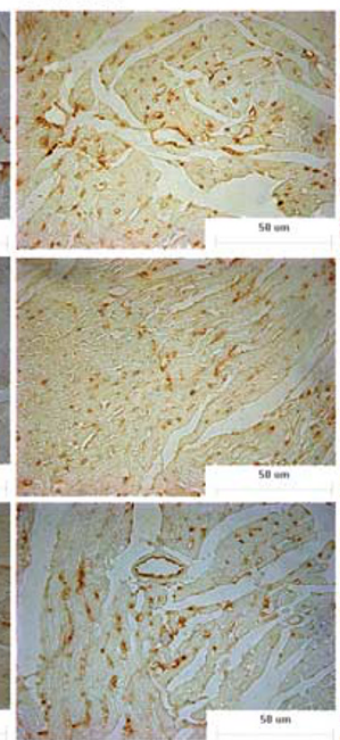

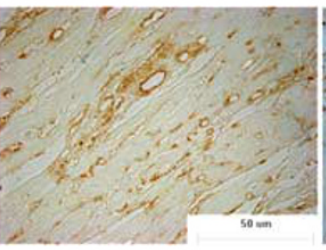

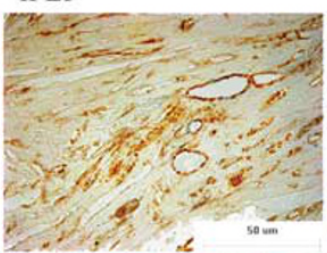

LYLI
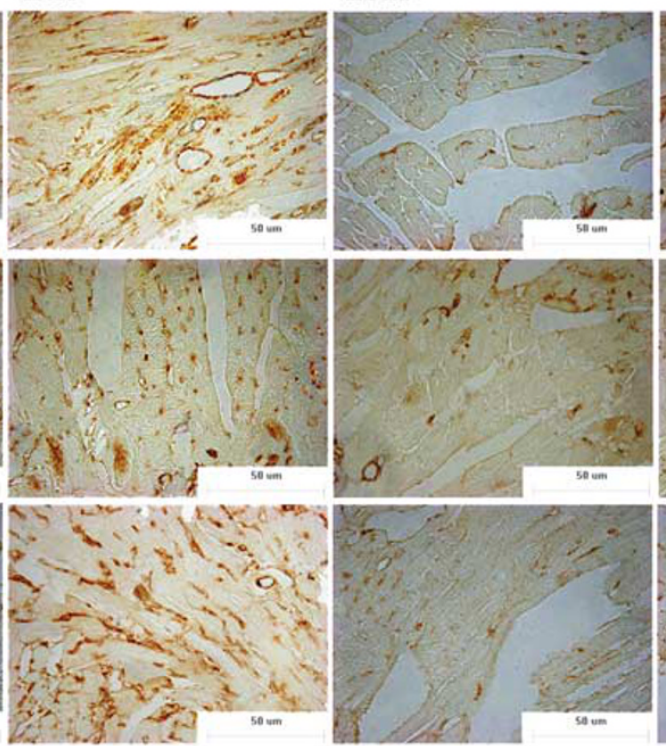

LI
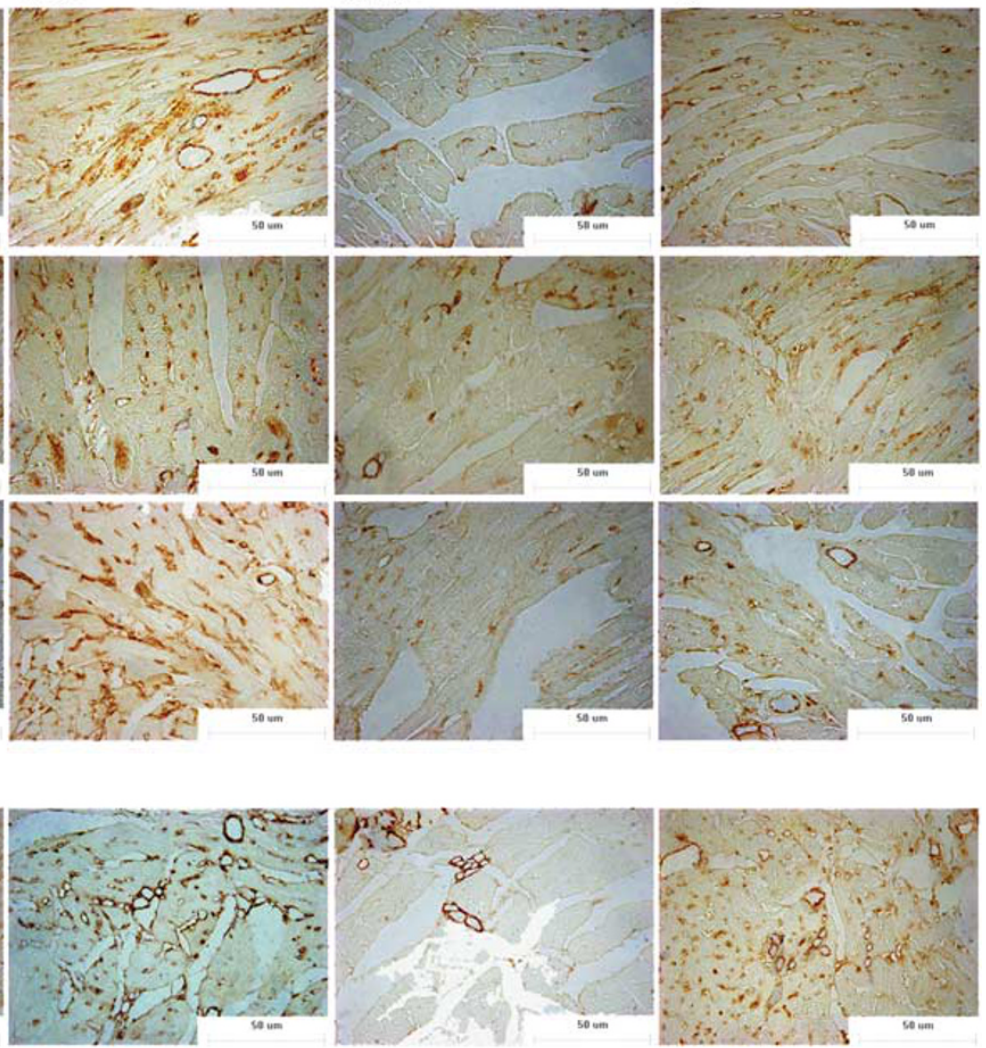

h

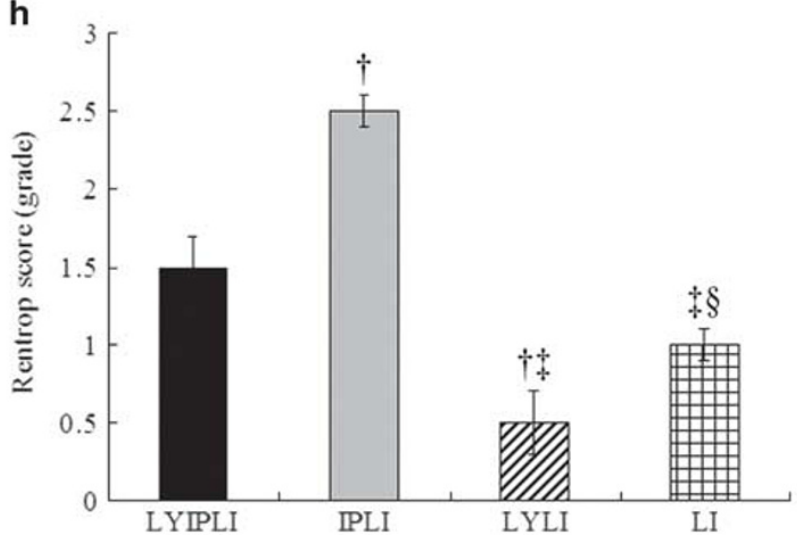

Figure 7 Continued.

by attenuating progressive myocardial remodeling and improving myocardial contractility. Similarly, AMI in LI pigs caused LV tissue remodeling including myocardial inflammation and bleeding (Figure 4), interstitial tissue edema, infarction, and collagen formation compared with the control group at 7 days after MI (Figure 5). PI3K inhibitor LY294002 injection aggravated these conditions in LYLI pig hearts; however, IP offset this effect maximally in IPLI pigs that received IP but not LY294002 injection before AMI induction. This suggests that the cardioprotective effect of IP protected against adverse left ventricular remodeling in pigs after MI, and the PI3K inhibitor LY294002 can diminish this cardioprotective effect, in agreement with the study of Elsherif et al $^{14}$ showing that PI3K inhibitors (wortmannin and LY294002) abolish the protection of IP.

\section{PI3K/Akt-Connexin 43}

The next step in this research was to elucidate the mechanism underlying the PI3K/Akt-antiarrhythmic effect of IP. Akt is an established mediator of IP in various species, ${ }^{15}$ and Akt activation directly results in $\mathrm{Cx} 43$ phosphorylation. ${ }^{16} \mathrm{Cx} 43$ is the major ventricular gap junction in the mammalian heart 
and numerous lines of evidence have demonstrated that gap junction-mediated intercellular coupling is essential for normal cardiac impulse propagation. For instance, Akt-dependent phosphorylation of Cx43 resulted in increased gap junction communication, providing some protection again lifethreatening cardiac arrhythmias. ${ }^{17}$ Indeed, this study showed that the expression levels of total Akt and phosphorylated Akt were higher at both the infarct zone and the border zone in pigs receiving IP treatment compared with pigs not receiving IP, and Cx43 expression revealed synchronous changes, consistent with the increase of viable myocardium in the infarct zone, and a reduction of malignant ventricular arrhythmia and pig death. However, injection of the PI3K inhibitor LY294002 diminished the PI3K/Akt-Cx43 cardioprotective effect induced by IP, and the expression level of total Akt, Akt phosphorylation, and $\mathrm{Cx} 43$ expression were lowest in the LYLI groups in pigs (Figure 6); this was consistent with the highest incidence of ventricular arrhythmias and pig death, and the smallest residual viable myocardium within the infarct zone. These findings suggest that increased expression and activation of both Akt and Cx43 induced by IP can contribute to a reduction of malignant ventricular arrhythmia. The mechanism by which the expression of connexin 43 was upregulated through PI3K/Akt by IP remains to be established. Whether PI3K/Akt is also required for $\mathrm{Cx} 43$ phosphorylation or saving the remaining myocardium within the infarction region in pigs is also unknown at present.

\section{PI3K/Akt-Mediated Angiogenesis}

The proangiogenic effect of IP as a result of increased VEGF production has also been reported by a number of studies evaluating infarcted myocardium. ${ }^{18}$ The involvement of other endogenous vascular permeabilizing factors, such as bFGF and Ang-1, certainly cannot be ruled out. ${ }^{19}$ In this study, we observed that at day 7 after MI, IP upregulated the expression levels of various proangiogenic growth factors, such as VEGF, as well as bFGF and Ang-1, compared with the control group and the groups not receiving IP. Similarly, IP also stimulated myocardial angiogenesis and collateral circulation in pigs subjected to MI (Figure 7). Our previous study demonstrated that intrinsic angiogenesis in the infarcted myocardium was correlated with local release of Akt through upregulation of VEGF, bFGF, and Ang- $1 .{ }^{10}$ In order to further investigate whether IP promoted this PI3K/Akt-mediated angiogenesis, the PI3K inhibitor LY294002 was injected into the myocardium before MI induction, leading to significantly reduced expression of Akt, Ang-1, bFGF, and VEGF, and vascular density and collateral circulation in the LYIPLI group. These negative effects of the PI3K inhibitor on angiogenesis and collateral circulation were the worst in LYLI pigs receiving LY294002 injection but not IP treatment. Therefore, IP appears to accelerate the process of intrinsic angiogenesis and collateral circulation during early myocardial ischemia via upregulation of PI3K/Akt-mediated proangiogenic factors. Our previous study also showed that the PI3-kinase/Akt pathway regulates proangiogenic gene transcription of VEGF, bFGF, and Ang-1, and in turn triggers Akt phosphorylation and activates Akt expression..$^{10}$ Recent in vitro studies showed that hypoxic preconditioning induces the expression of proangiogenic markers in stem cells, ${ }^{20}$ enhances survival of human adipose-derived stem cells, and conditions endothelial cells. $^{21}$ Whether these mechanisms in stem cells promote neovascularization in infarcted hearts warrants further investigation. This may be related to the regulatory feedback mechanism described in Supplementary Figure S2: IP activates the PI3K/Akt pathway, triggers Akt phosphorylation, promotes Akt expression, then upregulates the expression of growth factors Ang-1, bFGF, and VEGF, and Cx43, and inhibits the inflammatory reaction and collagen formation, resulting in enhancement of angiogenesis and collateral circulation, increase in cardiac function, improvement in heart remodeling, and reduction of malignant ventricular arrhythmia. Upregulated Ang-1, bFGF, and VEGF may in turn stimulate Akt phosphorylation. In contrast, LY294002 diminishes these effects, and LY294002 injection may lead to deterioration of heart failure and cardiac remodeling that ultimately deteriorated malignant ventricular arrhythmia and damaged cardiac repair.

LY294002 is a PI3K inhibitor, and has inhibitory effects on PI3K/Akt. However, it might also have other effects via PI3K inhibition. Inhibition of PI3K activity by LY294002 is generally known to induce apoptosis and inhibit cell growth. ${ }^{22}$ In some cases, LY294002 displays significant off-target effects on other kinases. ${ }^{23}$ In this study, the arrhythmia episode time and accumulation episode duration time in the LYLI group was larger than those in the LI group (Figure 2), suggesting that LY294002 itself has proarrhythmic effects. Therefore, further exploration into whether the inhibition of IP by LY294002 or the proarrhythmic effects of LY294002 are more predominantly involved in this inhibition is warranted. Although it is clear that LY294002 exerted significant effects on many of the above-mentioned situations, the effects of LY294002 on the survival function (Figure 1), malignant arrhythmia initial episode time (Figure 2), and LVEF, LVFS, LVEDIVST, and LVEDPWT (Figure 3) were similar between the LYIPLI group and the LYLI group, corresponding with the similar change of pSer473 between the LYIPLI group and the LYLI group (Figure 6), and this could mean that Akt has other effects independent of IP and myocardial infarction, such as autophagy, ${ }^{24}$ neuroprotective potential, ${ }^{25}$ and decreasing macrophage retention. ${ }^{26}$

Although LY294002 has been extremely useful and is still widely employed as a research probe, it has a very significant limitation: LY294002 is a weak inhibitor with only micromolar potency. ${ }^{23}$ Our study showed similar findings: in comparison with the LYLI group, fewer arrhythmia episodes and cumulative episode time (Figure 2b), smaller LVEDV, LVESV, LVEDD, and LVESD (Figure 3), lower inflammation (Figure 4), less infarct size (Figures $5 \mathrm{a}$ and $\mathrm{c}$ ), and collagen content (Figures $5 \mathrm{~b}$ and e), and greater viable myocardium (Figure 5d) were seen in the LYIPLI group, in accordance 
with higher expression of Akt, Cx43 (Figure 6), Ang-1, bFGF, and VEGF, more blood vascular density, and collateral circulation (Figure 7). This discrepancy implies that LY294002, acting as a PI3K inhibitor via incomplete inhibition of Akt phosphorylation, cannot completely abolish IP-mediated myocardial repair and antiarrhythmia. Furthermore, other passways beside Akt may be involved in antiarrhythmogenesis and cardiac function. For example, Jacobshagen et al ${ }^{27}$ suggested that inhibition of PI3K with wortmannin increases phosphorylation of phospholamban. Phospholamban is well known to be involved intracellular Ca handling, and hence its function is related to the arrhythmias and cardiac function. Other studies demonstrated that PI3K/heme oxygenase 1 (HO-1)-dependent antioxidant defensive system, ${ }^{28} \mathrm{PI} 3 \mathrm{~K}-$ kinase IA activation, ${ }^{29}$ and PI3K-mediated eNOS activation are critical for IP-induced cardioprotection. ${ }^{30}$

In conclusion, ischemic preconditioning reduces malignant ventricular arrhythmia and improves cardiac repair within AMI via activation of signal pathways of PI3K/Akt-mediated Cx43 and proangiogenic factors.

Supplementary Information accompanies the paper on the Laboratory Investigation website (http://www.laboratoryinvestigation.org)

\section{ACKNOWLEDGMENTS}

We thank Mr Renmin Yao (Shanghai Institute of Cardiovascular Diseases, Zhongshan Hospital, Fudan University, Shanghai, China) for technical assistance. This study was supported by grants from the National Natural Sciences Foundation of China (81170103 and 81270172 to S Zhang) and Shanghai Health Commission Scientific Research Fund Projects (20134334 to F Su).

\section{DISCLOSURE/CONFLICT OF INTEREST}

The authors declare no conflict of interest.

1. Rubart M, Zipes DP. Mechanisms of sudden cardiac death. J Clin Invest 2005;115:2305-2315.

2. Spear JF1, Moore EN. Preconditioning attenuates the shortening of recovery during coronary occlusion in isolated rabbit hearts with D-sotalol-induced long QT intervals. J Cardiovasc Pharmacol 2002;39: 761-776.

3. Zhou $\mathrm{XL}$, Wan $\mathrm{L}, \mathrm{Xu} \mathrm{QR}$ et al. Notch signaling activation contributes to cardioprotection provided by ischemic preconditioning and postconditioning. J Transl Med 2013;11:251.

4. Kaneko H1, Anzai T, Naito K et al. Role of ischemic preconditioning and inflammatory response in the development of malignant ventricular arrhythmias after reperfused ST-elevation myocardial infarction. J Card Fail 2009;15:775-781.

5. Wang $Y$, Huang CX, Zhou YF et al. ADAMTS-1 expression in rat myocardium after ischemic preconditioning: age-associated differences. Chin Med J (Engl) 2011;124:95-99.

6. Cuevas P, Carceller F, Giménez-Gallego G. Fibroblast growth factors in myocardial ischemia/reperfusion injury and ischemic preconditioning. J Cell Mol Med 2001;5:132-142.

7. Anzai T, Naito K, Kohno T et al. Cholesterol diet leads to attenuation of ischemic preconditioning-induced cardiac protection: the role of connexin 43. Am J Physiol Heart Circ Physiol 2011;300:H1907-H1913.

8. Cai ZP, Parajuli N, Zheng $X$ et al. Remote ischemic preconditioning confers late late protection against myocardial ischemia-reperfusion injury in mice by upregulating interleukin-10. Basic Res Cardiol 2012;107:277.
9. Dunn CA, Su V, Lau AF et al. Activation of Akt, not connexin 43 protein ubiquitination, regulates gap junction stability. J Biol Chem 2012;287: 2600-2607.

10. Zhang $S$, Zhao $L$, Shen $L$ et al. Comparison of various niches for endothelial progenitor cell therapy on ischemic myocardial repair: coexistence of host collateralization and Akt-mediated angiogenesis produces a superior microenvironment. Arterioscler Thromb Vasc Bio 2012;32:910-923.

11. Barra $S$, Providência R, Paiva $L$ et al. Early repolarization patterns and the role of additional proarrhythmic triggers. Europace 2013;15: 482-485.

12. Ledvenyiova V, Pancza D, Matejikov JJ et al. Impact of age and sex on response to ischemic preconditioning in the rat heart: differential role of the PI3K-AKT pathway. Can J Physiol Pharmacol 2013;91:640-647.

13. $\mathrm{Xu} \mathrm{WQ}, \mathrm{Yu} Z$, Xie $\mathrm{Y}$ et al. Therapeutic effect of intermittent hypobaric hypoxia on myocardial infarction in rats. Basic Res Cardiol 2011;106: 329-342.

14. Elsherif L, Wang X, Grachoff $M$ et al. Cardiac-specific expression of the tetracycline transactivator confers increased heart function and survival following ischemia reperfusion injury. PLoS One 2012;7: e30129.

15. Yang X, Cohen MV, Downey JM. Mechanism of cardioprotection by early ischemic preconditioning. Cardiovasc Drugs Ther 2010;24: 225-234.

16. Batra N, Riquelme MA, Burra $S$ et al. Direct regulation of osteocytic connexin 43 hemichannels through AKT kinase activated by mechanical stimulation. J Biol Chem 2014;289:10582-10591.

17. Kim E, Fishman Gl. Designer gap junctions that prevent cardiac arrhythmias. Trends Cardiovasc Med 2013;23:33-38.

18. Lim SY, Hsiao ST, Lokmic Z et al. Ischemic preconditioning promotes intrinsic vascularization and enhances survival of implanted cells in an in vivo tissue engineering model. Tissue Eng Part A 2012;8:2210-2219.

19. Nagy JA, Benjamin L, Zeng $\mathrm{H}$ et al. Vascular permeability, vascular hyperpermeability and angiogenesis. Angiogenesis 2008;11:109-119.

20. Chacko SM, Ahmed S, Selvendiran K et al. Hypoxic preconditioning induces the expression of prosurvival and proangiogenic markers in mesenchymal stem cells. Am J Physiol Cell Physiol 2010;299: C1562-C1570.

21. Stubbs SL, Hsiao ST, Peshavariya HM et al. Hypoxic preconditioning enhances survival of human adipose-derived stem cells and conditions endothelial cells in vitro. Stem Cells Dev 2012;21:1887-1896.

22. Chen J. Roles of the PI3K/Akt pathway in Epstein-Barr virus-induced cancers and therapeutic implications. World J Virol 2012;1:154-161.

23. Workman P, Clarke PA, Raynaud Fl et al. Drugging the PI3 kinome: from chemical tools to drugs in the clinic. Cancer Res 2010;70:2146-2157.

24. Wu X, He L, Cai $Y$ et al. Induction of autophagy contributes to the myocardial protection of valsartan against ischemia-reperfusion injury. Mol Med Rep 2013;8:1824-1830.

25. Ye Z, Wang N, Xia $\mathrm{P}$ et al. Delayed administration of parecoxib, a specific COX-2 inhibitor, attenuated postischemic neuronal apoptosis by phosphorylation Akt and GSK-3ß. Neurochem Res 2012;37:321-329.

26. Li X, Mikhalkova D, Gao E et al. Myocardial injury after ischemiareperfusion in mice deficient in Akt2 is associated with increased cardiac macrophage density. Am J Physiol Heart Circ Physiol 2011;301: H1932-H1940.

27. Jacobshagen C, Kortlepel S, Unsoeld BW et al. Inhibition of PI3K improves contractility in alpha1-adrenergically stimulated myocardium. Front Biosc 2008;13:6841-6849.

28. Yin $X$, Wang $X$ et al. Hyperbaric oxygen preconditioning attenuates myocardium ischemia-reperfusion injury through upregulation of heme oxygenase 1 expression: PI3K/Akt/Nrf2 pathway involved. J Cardiovasc Pharmacol Ther 2015 [e-pub ahead of print].

29. Duan $Q$, Madan ND, Wu J et al. Role of phosphoinositide 3-kinase IA (PI3K-IA) activation in cardioprotection induced by ouabain preconditioning. J Mol Cell Cardiol 2015;80:114-125.

30. Yang C, Talukder MA, Varadharaj $S$ et al. Early ischaemic preconditioning requires Akt- and PKA-mediated activation of eNOS via serine1176 phosphorylation. Cardiovasc Res 2013;97:33-43. 